\title{
Mucoadhesive maleimide-functionalised liposomes for drug delivery to urinary bladder
}

Article

Accepted Version

Creative Commons: Attribution-Noncommercial-No Derivative Works 4.0

Kaldybekov, D., Tonglairoum, P., Opanasopit, P. and Khutoryanskiy, V. V. (2018) Mucoadhesive maleimidefunctionalised liposomes for drug delivery to urinary bladder. European Journal of Pharmaceutical Sciences, 111. pp. 83-90. ISSN 0928-0987 doi:

https://doi.org/10.1016/j.ejps.2017.09.039 Available at https://centaur.reading.ac.uk/72941/

It is advisable to refer to the publisher's version if you intend to cite from the work. See Guidance on citing.

Published version at: http://www.sciencedirect.com/science/article/pii/S0928098717305316

To link to this article DOI: http://dx.doi.org/10.1016/j.ejps.2017.09.039

Publisher: Elsevier

All outputs in CentAUR are protected by Intellectual Property Rights law, including copyright law. Copyright and IPR is retained by the creators or other copyright holders. Terms and conditions for use of this material are defined in the End User Agreement. 


\section{CentAUR}

Central Archive at the University of Reading

Reading's research outputs online 
${ }^{a}$ School of Pharmacy, University of Reading, Whiteknights, Reading, RG6 6AD, United Kingdom

${ }^{b}$ Faculty of Chemistry and Chemical Technology, Al-Farabi Kazakh National University, Almaty, 050040, Kazakhstan

${ }^{c}$ Pharmaceutical Development of Green Innovations Group, Faculty of Pharmacy, Silpakorn University, Nakhon Pathom, 73000, Thailand

*Corresponding author:

13 Postal address: School of Pharmacy, University of Reading, Whiteknights, Reading, RG6 6AD, United 14 Kingdom

15 E-mail address: v.khutoryanskiy@ reading.ac.uk

16 Telephone: $+44(0) 1183786119$

Fax: +44 (0) 1183784703

Abstract

Intravesical drug administration is used to deliver chemotherapeutic agents via a catheter to treat bladder cancer. The major limitation of this treatment is poor retention of the drug in the bladder due to periodic urine voiding. In this work, maleimide-functionalised PEGylated liposomes (PEG-Mal) were explored as mucoadhesive vehicles for drug delivery to the urinary bladder. The retention of these liposomes on freshly excised porcine bladder mucosa in vitro was compared with conventional liposomes, PEGylated liposomes, two controls (dextran and chitosan), and evaluated through Wash 26 Out $_{50}\left(\mathrm{WO}_{50}\right)$ values. PEG-Mal liposomes exhibited greater retention on mucosal surfaces compared to other liposomes. The penetration abilities of conventional, PEG-Mal-functionalised and PEGylated liposomal dispersions with encapsulated fluorescein sodium into the bladder mucosa ex vivo were assessed using a fluorescence microscopy technique. PEGylated liposomes were found to be more mucosa-penetrating compared to other liposomes. All liposomes were loaded with fluorescein sodium salt as a model drug and the in vitro release kinetics was evaluated. Longer drug release was observed from PEG-Mal liposomes. 
Keywords: liposomes, urinary bladder, intravesical drug delivery, mucoadhesion, mucus penetration, wash out $50\left(W_{50}\right)$

\section{Introduction}

Bladder cancer (BC) is caused by uncontrolled growth of tumour cells in the urinary bladder. It has the 9th highest incidence globally, with an estimated 430,000 newly diagnosed cases in 2012 (Stewart and Wild, 2014). The prevalence of this malignancy of the genitourinary tract tends to increase with economic development and males are more likely to develop this condition than females (Torre et al., 2015). The most common type of BC is transitional cell carcinomas that comprise over $90 \%$ of tumours, while squamous cell carcinomas and adenocarcinomas represent about $5 \%$ and $1 \%$ of the reported cases, respectively.

Intravesical drug delivery (IDD) is a direct administration of therapeutic agents into the bladder via insertion of a urethral catheter (Au et al., 2001; Malmström, 2003; Kolawole et al., 2017). This allows localised treatment, minimises adverse effects and improves the exposure of the diseased tissues to therapeutic agents. Also, the oral route of the drug intake is undesirable in the therapy of $\mathrm{BC}$ due to absorption, metabolism and renal excretion, resulting in poor drug bioavailability in the bladder.

IDD has intrinsic limitations related to the substantial chemotherapy dilution and wash out due to urinary voiding, low permeability of the urothelium, and intermittent catheterisations (GuhaSarkar and Banerjee, 2010). Additionally, the procedure is relatively unpleasant for the patients and may cause inflammatory reactions and infections. To counteract the limitations associated with low drug permeability, mucoadhesive formulations offer great promise. The ability of mucoadhesive materials to adhere to the bladder epithelium and withstand wash out effect could improve drug bioavailability by prolonging the residence in the bladder. Mucoadhesive formulations for IDD must fulfill the following criteria: the dosage form should have rapid and efficient adhesion to the bladder mucosa; must not interfere with the normal physiology of the bladder; and should be able to stay adhered in situ for a few hours even after urination (Tyagi et al., 2006).

A number of mucoadhesive formulations have been researched, including the use of hydrophilic polymers of both natural and synthetic type, such as chitosan, carbomers and cellulose derivatives (Hombach and Bernkop-Schnürch, 2010; Khutoryanskiy, 2011). The adherence of these polymers is due to the ability to interact with mucin glycoproteins via non-covalent bonds such as hydrogen bonds, electrostatic interactions and chain entanglements, diffusion and interpenetration (Khutoryanskiy, 
exhibit greater mucoadhesion to pig vesical mucosa compared to carboxymethylcellulose and polycarbophyl, thus resulting in a slower drug release and longer residence time (Burjak et al., 2001).

In recent years, various chemical approaches have been used to improve mucoadhesive properties of polymers by introducing specific functional groups such as thiols (Bernkop-Schnürch, 2005; Davidovich-Pinhas et al., 2009; Cook et al., 2015), acrylates (Davidovich-Pinhas and Bianco-Peled, 2011; Brannigan and Khutoryanskiy, 2017), and catechols (Kim et al., 2015). Some studies reported the use of chemically modified mucoadhesive materials for IDD to urinary bladder. Thiol-modified chitosan nanoparticles (NPs) have been used for IDD in an in vitro study using porcine urinary bladder (Barthelmes et al., 2011). It was found that chitosan functionalised with thiol groups demonstrated superior mucoadhesion, greater stability and controlled release compared to the unmodified chitosan NPs. In a different study, the retention of thiolated chitosan NPs on rat bladder mucosa in vivo was approximately 170-fold greater compared to the polymer-free fluorescent marker (Barthelmes et al., 2013). Mun et al. (2016) developed and evaluated the retention of thiolated and PEGylated silica NPs on porcine urinary bladder mucosa in vitro through use of a novel Wash Out 50 (WO $\left.\mathrm{W}_{50}\right)$ quantitative method. It was shown that the retention of these NPs on bladder mucosa depended on both their thiol content and dimensions.

Recently we have demonstrated for the first time that polymers functionalised with maleimide groups exhibit excellent mucoadhesive properties to conjunctival tissues ex vivo and the ability of these materials to retain on mucosal tissues was comparable to well-known mucoadhesive chitosan (Tonglairoum et al., 2016). This excellent mucoadhesive performance of maleimide-functionalised polymers is due to their ability to form covalent linkages with thiol-groups present in mucins. More recently, Shtenberg et al. (2017) reported the functionalisation of alginate with maleimide-terminated polyethyleneglycol to achieve superior mucoadhesive properties towards porcine intestine mucosa.

Liposomes are microscopic vesicles composed of phospholipid bilayers with the size range from $30 \mathrm{~nm}$ up to several microns that have attracted a lot of interest over the past four decades as pharmaceutical carriers. Conventional liposomes and liposomes coated with mucoadhesive polymers previously were used for transmucosal drug delivery (Sasaki et al., 2013; Berginc et al., 2014; Adamczak et al., 2017). Some liposome-based formulations were also reported for intravesical drug delivery (Chuang et al., 2009, 2014; Kawamorita et al., 2016). Recently, Oswald et al. (2016) reported the preparation and characterisation of maleimide-functionalised liposomes; however they did not demonstrate any application of these systems for drug delivery.

In this study, we explored the mucoadhesive properties of maleimide-functionalised liposomes and compared their retention on urinary bladder mucosa with conventional liposomes and PEGylated 
liposomes. We also have studied the physicochemical properties of different liposomes, their

100 penetration into the bladder mucosa and drug release profiles.

101

102

\section{Materials and methods}

103

\subsection{Materials}

Soybean L-alpha-phosphatidylcholine (PC) was purchased from Alfa Aesar (Heysham, UK). [N(carbonyl-methoxypolyethylene glycol-2000)-1,2-distearoyl-sn-glycero-3-phosphoethanol-amine, sodium salt] (MPEG $\left.{ }_{2000}-\mathrm{DSPE}\right)$ was a generous gift from Lipoid GmbH (Ludwigshafen, Germany). 1,2-distearoyl-sn-glycero-3-phosphoethanolamine- $\mathrm{N}$-[maleimide(polyethylene glycol)-2000] ammonium salt ( $\mathrm{PEG}_{2000}$-DSPE-Mal) was purchased from Avanti Polar Lipids (Alabaster, USA). Cholesterol (CHO), chitosan (low molecular weight; Mw $62.3 \mathrm{kDa}$, PDI 3.42 as reported by Symonds et al (2016)), fluorescein isothiocyanate dextran (FITC-dextran, $\mathrm{M}_{\mathrm{w}}$ 3000-5000 Da), fluorescein isothiocyanate (FITC) and fluorescein sodium salt (NaFI) were purchased from Sigma Aldrich (Gillingham, UK). All other chemicals were of analytical grade and were used as received. $0.24 \mathrm{~g} \mathrm{KH}_{2} \mathrm{PO}_{4}$ ( $\mathrm{pH} 7.4$ ). The buffer solution was made with deionised water (total volume $1000 \mathrm{~mL}$ ).

\subsection{Preparation of liposomes}

The liposomal formulations containing fixed amounts of PC, CHO and PEGylated lipids at molar ratios of 10:2:0 and 10:2:3 $\mathrm{mM}$ (Table 1) were prepared using thin film hydration and sonication method (Rangsimawong et al., 2016). In brief, a mixture of PC, CHO and PEGylated lipids dissolved in chloroform-methanol $(2: 1, \mathrm{v} / \mathrm{v})$ in test tubes. The organic solvent was evaporated under a stream of nitrogen and a thin film of lipid was formed inside the test tubes. The test tubes were then placed under vacuum at least $6 \mathrm{~h}$ to remove any residual solvent. Then, solution of NaFI in PBS ( $\mathrm{pH}$ 7.4) was added to the dried lipid films to generate hydrated liposome vesicles and the tubes were left for $1 \mathrm{~h}$ at room temperature. The tubes were vortex-mixed vigorously for $30 \mathrm{~min}$ and these liposome dispersions were then sonicated in a sonication bath (FS200b, Decon Laboratories Ltd., UK) for $30 \mathrm{~min}$ to reduce the size of the liposomes. Excess lipids were separated from the vesicle formulations by centrifugation at $14000 \mathrm{rpm}(8765 \times \mathrm{g})$ at $4{ }^{\circ} \mathrm{C}$ for $30 \mathrm{~min}$. The supernatants were collected and stored in a fridge overnight prior to characterisation.

\subsection{Synthesis of fluorescently-labelled chitosan}


FITC-chitosan was synthesised according to the procedure described previously (Cook et al.,

132 2011; Symonds et al., 2016). Briefly, $1 \mathrm{~g}$ of chitosan was dissolved in $100 \mathrm{~mL}$ of acetic acid (0.1 M)

133 and left stirring overnight. $100 \mathrm{mg}$ of FITC was dissolved in $50 \mathrm{~mL}$ of methanol and subsequently was

134 added to the chitosan solution and stirred for $3 \mathrm{~h}$ in the dark at room temperature. The modified

135 chitosan was then precipitated in $1 \mathrm{~L}$ of $0.1 \mathrm{M}$ sodium hydroxide and filtered. The resulting product

136 was redissolved and purified by dialysis against deionised water in the dark to remove any unreacted

137 FITC before lyophilisation. FITC-chitosan was kept wrapped in aluminum foil to avoid exposure to

138 light and stored in a fridge for further use.

\subsection{Particle size and zeta potential analysis}

141 The size of liposomes, their polydispersity index (PDI) and zeta potential values were determined 142 using dynamic light scattering (DLS) with a Zetasizer Nano-ZS (Malvern Instruments, UK). Each 143 formulation was diluted 100-fold with ultrapure water. A typical liposome refractive index of 1.45 and 144 absorbance of 0.1 were used in all measurements. Each sample was analysed three times at $25^{\circ} \mathrm{C}$ and 145 the mean \pm standard deviation values were calculated.

\subsection{Transmission electron microscopy}

148 TEM images were generated using a JEOL 2100Plus TEM operating at an acceleration voltage of $149200 \mathrm{kV}$. Specimens were prepared by pipetting a drop of liposome suspension diluted with water (about $1505 \mathrm{mg} / \mathrm{mL}$ ) onto a parafilm. A glow-discharged holey carbon film-coated 400-mesh copper grid was 151 then placed onto the drop with "carbon" side and left in contact with the sample for 1 min. The excess 152 solution was removed by blotting with a filter paper. The grid was washed by touching its surface with sample side down on drop of deionised water on parafilm for $1 \mathrm{~min}$ and then blotted dry with a filter

154 paper. A drop of $1 \%(\mathrm{w} / \mathrm{v})$ uranyl acetate (UA) solution was applied on parafilm and the grid remained 155 in contact with UA for $30 \mathrm{sec}$ (PEG-Mal liposomes were stained for $5 \mathrm{sec}$, which provided better quality of TEM images). The excess stain was removed by dabbing similarly and the sample was left to 157 dry in air prior to TEM characterisation.

\subsection{Encapsulation efficacy and loading capacity}

The lipid nanocarrier dispersion $(500 \mu \mathrm{L})$ was placed in an ultrafiltration tube using an Amicon® Ultra-0.5 Ultracel-3 centrifugal filter unit with a molecular weight cutoff of $3 \mathrm{kDa}$ and centrifuged at 4

$162{ }^{\circ} \mathrm{C}$ at $14000 \mathrm{rpm}(8765 \times \mathrm{g})$ for $60 \mathrm{~min}$. The filtrate was discarded, and $250 \mu \mathrm{L}$ of PBS was added 163 before further centrifugation at $4{ }^{\circ} \mathrm{C}$ at $14000 \mathrm{rpm}(8765 \times \mathrm{g})$ for $40 \mathrm{~min}$. This washing step was 
repeated twice. The NaFI-loaded liposomes in the retentate were then disrupted with $200 \mu \mathrm{L}$ of methanol and centrifuged at $4{ }^{\circ} \mathrm{C}$ at $14000 \times \mathrm{g}$ for $10 \mathrm{~min}$. The amount of free $\mathrm{NaFI}$ in the supernatants was quantified using a Varian Cary Eclipse fluorescence spectrometer at $\lambda_{\text {excitation }}$ and $\lambda_{\text {emission }}=460$ and $512 \mathrm{~nm}$, respectively, and the encapsulation efficiency (\%EE) and loading capacity (\%LC) were calculated using the following equations:

$$
\begin{gathered}
\% E E=\frac{C}{C_{i}} \times 100 \\
\% L C=\frac{C}{\text { Lipid composition }} \times 100
\end{gathered}
$$

where $\mathrm{C}$ is the amount of $\mathrm{NaFI}$ entrapped in the liposomes, and $\mathrm{C}_{\mathrm{i}}$ is the initial amount of NaFI.

A calibration curve used to calculate the encapsulation efficacy and loading capacity can be found in 175 Supplementary Information (Fig S1).

176

\subsection{Preparation of artificial urine solution}

Artificial urine solution was prepared according to the previously reported procedure (Chutipongtanate and Thongboonkerd, 2010). The following components were dissolved in deionised water by stirring for $6 \mathrm{~h}$ at room temperature, before making the total volume to $2000 \mathrm{~mL}$ : urea (24.27 g), uric acid $(0.34 \mathrm{~g})$, creatinine $(0.90 \mathrm{~g}), \mathrm{Na}_{3} \mathrm{C}_{6} \mathrm{H}_{5} \mathrm{O}_{7} \cdot 2 \mathrm{H}_{2} \mathrm{O}(2.97 \mathrm{~g}), \mathrm{NaCl}(6.34 \mathrm{~g}), \mathrm{KCl}(4.50 \mathrm{~g})$, $\mathrm{Na}_{2} \mathrm{SO}_{4}(2.58 \mathrm{~g}), \mathrm{NaH}_{2} \mathrm{PO}_{4} \cdot \mathrm{H}_{2} \mathrm{O}(1.00 \mathrm{~g})$, and $\mathrm{Na}_{2} \mathrm{HPO}_{4}(0.11 \mathrm{~g})$. The artificial urine solution (pH 6.4) was kept at $37^{\circ} \mathrm{C}$ throughout the experiments.

\subsection{In vitro retention studies on porcine urinary bladder}

The retention of the liposomes on porcine urinary bladder tissues were determined using a protocol slightly modified from Mun et al. (2016). Porcine bladder tissues were received from P.C. Turner Abattoirs (Farnborough, UK), immediately after animal slaughter, packed with dry ice and transported in a polystyrene container. The tissues were defrosted upon arrival and carefully excised to yield approximately $2 \times 3 \mathrm{~cm}$ sections, avoiding contact with the internal mucosa, which were then used in the experiments. The dissected bladder tissue was mounted on a glass slide with mucosal side facing upward and rinsed with $3 \mathrm{~mL}$ of AU solution. Experiments were performed with the bladder tissues maintained at $37^{\circ} \mathrm{C}$ in an incubator. Aliquots from NaFI-loaded liposome stock solutions were 
withdrawn and diluted 1:1 with PBS $(2.3 \mathrm{mg} / \mathrm{mL})$, and aqueous solutions of FITC-chitosan $(0.5 \mathrm{mg} / \mathrm{mL}$ in $0.5 \%$ acetic acid) and FITC-dextran $\left(0.5 \mathrm{mg} / \mathrm{mL}\right.$ in deionised $\left.\mathrm{H}_{2} \mathrm{O}\right)$ were prepared. The $\mathrm{pH}$ of FITCchitosan solution was adjusted to $\mathrm{pH} 6$ with $1 \% \mathrm{NaOH}$. An aliquot $(20 \mu \mathrm{L})$ of either NaFI-loaded lipid nanocarrier dispersions or polymers (controls) was pipetted onto a mucosal surface and irrigated with AU solution at a flow rate of $2 \mathrm{~mL} / \mathrm{min}$ using a syringe pump (total washing time was $50 \mathrm{~min}$ ). Fluorescence images of whole tissue were taken using a Leica MZ10F stereo-microscope (Leica Microsystems, UK) with Leica DFC3000G digital camera at $0.8 \times$ magnification with $20 \mathrm{~ms}$ exposure time, fitted with a GFP filter. The microscopy images were then analysed with ImageJ software by measuring the pixel intensity after each wash. The pixel intensity of the blank samples (bladder mucosa without test material) were subtracted from each measurement. Each experiment was conducted in triplicate.

Evaluation of retention of formulations on the mucosa in vitro was quantified through $\mathrm{WO}_{50}$ values, which represent the volume of a biological fluid necessary to wash out 50\% of a mucoadhesive formulation from a substrate (Mun et al., 2016). $\mathrm{WO}_{50}$ values of test materials were calculated via extrapolation of the wash-off profiles to $50 \%$ using polynomial fitting.

\subsection{Mucosal penetration}

The mucosal permeation study was carried out as described in Mansfield et al. (2016) using freshly excised porcine bladder tissues. NaFI-loaded liposome solutions were diluted 1:1 with PBS. Aliquots $(100 \mu \mathrm{L})$ of NaFI-loaded liposomes were deposited onto $2 \times 2 \mathrm{~cm}^{2}$ ex vivo bladder mucosa, which were then placed on microscope slides. Deionised water was also pipetted as a blank control. Samples were left to incubate for $15,30,45$ and $60 \mathrm{~min}$ at $37{ }^{\circ} \mathrm{C}$. Following each time point, tissue pieces were placed with mucosal layer facing upward into a weighing boat $(3.5 \times 5.5 \mathrm{~cm})$, half filled with OCT, a cryoprotective embedding medium. They were then placed on dry ice, before being completely embedded in OCT to conserve the liposome-loaded mucus membrane. Samples were then left on dry ice for $3 \mathrm{~h}$.

For sectioning, each sample was mounted onto a $22 \mathrm{~mm}$ standard metal sample holder using OCT, and placed on dry ice for 30 min until completely frozen. Mucosal tissues were cryosectioned transversely with a standard $189 \times 27 \times 10 \mathrm{~mm}$ blade at $5^{\circ}$ to form $25 \mu \mathrm{m}$ sections, placed onto Superfrost ${ }^{\circledR}$ Plus charged microscope slides (Thermo Scientific, UK) and left to dry in air for $30 \mathrm{~min}$ before being stored. All sections were cut upwards through the mucosal layer. All specimens were cut using a Bright 5040 cryostat in a Bright Model PTF freezing chamber at $-25^{\circ} \mathrm{C}$ (Bright Instrument Co. Ltd, UK). 
Sections were placed under the Leica MZ10F fluorescence stereo-microscope and all images taken with $160 \mathrm{~ms}$ exposure time through the GFP filter. 10 images were taken for each liposome type 230 from a separate section of tissue.

231 Image J software was used to evaluate penetration of the liposomes as described by Mansfield et 232 al. (2016). For each image, the background was subtracted, a line drawn across the mucosal barrier, and 233 the "plot profile" measured. This was repeated five times at random locations along the mucosal 234 surface for each image, giving 50 profiles for each sample. These profiles were then evaluated for 235 penetration of liposomes. This was achieved by measuring the widths of all peaks including the width 236 of the last peak as the urinary bladder mucosa is heavily folded. The mean values were calculated 237 following analysis of each profile. To determine penetration into mucosa the values obtained for the blank tissue at each time point were then subtracted from the other values at the same time point.

\subsection{In vitro release of NaFI from liposomes}

The in vitro release of NaFI from liposomes was studied using a dialysis method adopted from our previous publication (Tonglairoum et al., 2016). In brief, $2 \mathrm{~mL}$ of NaFI-loaded liposomes in AU solution was transferred in a Pur-A-Lyzer ${ }^{\mathrm{TM}}$ Maxi 3500 dialysis membrane and immersed in $30 \mathrm{~mL}$ of $\mathrm{AU}\left(\mathrm{pH}\right.$ 6.4) that was then shaken at $80 \mathrm{spm}$ for $24 \mathrm{~h}$ at $37^{\circ} \mathrm{C}$. At regular intervals, aliquots $(5 \mathrm{~mL})$ were withdrawn from the dialysate and replaced with fresh medium to maintain a constant volume. The released $\mathrm{NaFI}$ was determined using fluorescence spectrometer $\left(\lambda_{\text {excitation }}=460\right.$ and $\left.\lambda_{\text {emission }}=512 \mathrm{~nm}\right)$. Fig 2S (Supplementary Information) shows the calibration curve used in these experiments. All release experiments were conducted in triplicate.

\subsection{Statistical analysis}

Statistical analysis was performed using GraphPad Prism, v5.0. Mean values \pm standard deviations were calculated and assessed for significance using one-way analysis of variance (ANOVA) 254 followed by Bonferoni post hoc test, where $\mathrm{p}<0.05$ was fixed as the statistical significance criterion.

\section{Results and discussion}

\subsection{Preparation and characterisation of liposomes}

259 The average mean diameter of all liposome preparations was $\sim 90 \pm 1 \mathrm{~nm}$ and the polydispersity index 260 (PDI) was less than 0.23, which indicates the presence of a homogeneous liposomal population with a 
narrow size distribution (Figure 1). The PDI is a measure of the size distribution and according to the 262 literature, liposomal formulation is considered to be homogenous if PDI is $\leq 0.30$ (Verma et al., 2003).

Vesicles showing their zeta potential of less than $-30 \mathrm{mV}$ are believed to have excellent colloidal stability and have the reduced number of bilayer membranes due to the electrostatic repulsion between the charges of the same polarity. Furthermore, liposomal formulation with $\leq-30 \mathrm{mV}$ would have higher entrapment capacity because stronger zeta potential contributes to the increase in the unilamellar vesicles (Sou, 2011; Kandzija and Khutoryanskiy, 2017). The physicochemical characteristics of different liposomes are summarised in Table 2.

Many factors influence the encapsulation efficiency (\%EE) and loading capacity (\%LC) of liposomes, including partition coefficient of the drug $(\log \mathrm{P})$, drug/liposome ratio, lipid composition, bilayer rigidity, presence of charge, method of preparation, etc (Kulkarni et al., 1995; Nii and Ishii, 2005). According to the literature water-soluble drugs have, however, lower encapsulation in the liposomes compared to their lipophilic counterparts (Kandzija and Khutoryanskiy, 2017); this depends on the encapsulated aqueous volume. Since NaFI has a $\log \mathrm{P}=-0.67$, we anticipated lower encapsulation levels (Nii and Ishii, 2005).

NaFI was used as a model drug to demonstrate the potential use of liposomes for the application in urinary bladder drug delivery. NaFI was loaded into the liposome formulations using standard thin film method followed by sonication. It was found that conventional liposomes had the highest \%EE

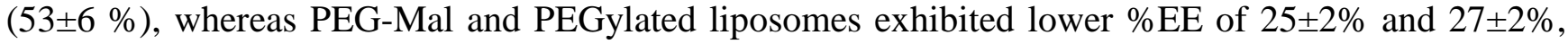
respectively (Table 2). It should be noted that \%EE values determined in the present study are not fully accurate as it was assumed that all $100 \%$ of lipids used in the formulation were converted into 282 liposomes.

Transmission electron microscopy (TEM) can be used to evaluate the morphology and fine 284 285 structure of liposomes. The freeze-fracture electron microscopy and/or cryo-electron microscopy are the optimal techniques to study the structure of rapidly frozen biological samples, membranes, proteins, etc. by TEM, but the preparation of the specimens (cryofixation, fracturation, vitrification and the following procedure of shading with evaporated platinum or gold, etc.) is complicated and requires long time (Frederik and Hubert, 2005; Robenek and Severs, 2008; Thompson et al., 2016). In our experience, negative staining is an easier and faster procedure. During negative staining liposomes are treated with an electron dense material achieving reasonable contrast. In this work, we used uranyl acetate that binds the phosphate group of phospholipids and has a limited penetration into the lipidic bilayer (Harris, 1986). 
TEM microphotographs of the produced liposomes are shown in Figure 2. TEM analysis revealed

294 the formation of a spherical and small unilamellar membrane for all liposome samples. Also, the 295 images show a population of homogeneous vesicles. It is also possible to see close bilayer structures spaced by free internal structure. Furthermore, the negative staining of these liposomes confirms the results obtained by the DLS analysis (Table 2). This observation is in agreement with the mechanism that the negative charge on the membrane increases the unilamellar vesicles that have high entrapment capacity. In addition, unlike conventional liposomes (Figure 2A), formation of aggregates can be observed in PEG-Mal liposome formulations, which is likely to be due to the hydrophobic nature of maleimide groups in their structure (Figure 2C).

302

\subsection{Mucoadhesion studies}

The retention properties of NaFI-loaded conventional, PEG-Mal and PEGylated liposomes on porcine urinary bladder mucosa were assessed using a flow-through method with fluorescent detection using the methodologies described in our previous publications (Irmukhametova et al., 2011; Storha et al., 2013; Mun et al., 2016). Figure 3 shows exemplary fluorescent images of the retention of conventional, PEG-Mal and PEGylated liposomal dispersions as well as two controls (chitosan and dextran) on urinary bladder mucosa, washed with artificial urine (AU). FITC-chitosan and FITCdextran were used as a positive and negative controls, respectively (Mun et al., 2016; Tonglairoum et al., 2016). However, it should be noted that there is a difference between the retention of FITCmodified polymers and retention of free sodium fluorescein released from liposomes. After analysis of the fluorescent images using ImageJ software, it was established that PEG-Mal liposomes exhibited very good mucoadhesive properties, comparable to the retention of FITC-chitosan (Figure 4). It was found that $32 \%$ of PEG-Mal liposomes remained on the bladder mucosa even after 50 min of washing with a total AU volume of $100 \mathrm{~mL}$. Moreover, the percentage retention of PEG-Mal liposomes was found not to be significantly different from FITC-chitosan ( $p>0.05$ ), confirming that PEG-Mal liposomes can also be adhered well on the bladder mucosa by forming covalent bonds with thiol groups present in mucin layer of the bladder epithelium. Conventional liposomes had a significantly lower retention capability compared to PEG-Mal liposomes ( $\mathrm{p}<0.05)$. It was found that approximately $18 \%$ of conventional liposomes retained on the bladder epithelial mucosa after $100 \mathrm{~mL}$ of washing with AU. These results confirm the mucoadhesive properties of maleimide-terminated PEGylated liposomes, which could also be used as a potential mucoadhesive drug carrier. The mechanism of enhanced mucoadhesion of maleimide-functionalised liposomes includes the formation of covalent linkages between maleimide groups and thiols present on mucosal surfaces, as shown in Figure 5. 
Mun et al. (2016) have described a novel quantitative method that allows evaluating and comparing the retention efficiency of liquid formulations on mucosal surfaces through the use of Wash Out $_{50}\left(\mathrm{WO}_{50}\right)$ values, which represent the volume of a biological fluid required to wash out $50 \%$ of the test mucoadhesive material from a substrate. In this work, $\mathrm{WO}_{50}$ values were calculated by analysing individual wash-off profiles and the results are summarised in Table 2. By comparing these values for different liposomes used in this study, it is clear that the PEG-Mal liposomes have greater retention on bladder mucosa $\left(\mathrm{WO}_{50}=48 \mathrm{~mL}, \mathrm{R}^{2}=0.9988\right)$, compared to conventional liposomes $\left(\mathrm{WO}_{50}=15 \mathrm{~mL}\right.$, $\left.\mathrm{R}^{2}=0.9987\right)$, PEGylated liposomes $\left(\mathrm{WO}_{50}=24 \mathrm{~mL}, \mathrm{R}^{2}=0.9985\right)$ and non-mucoadhesive FITC-dextran $\left(\mathrm{WO}_{50}=5 \mathrm{~mL}, \mathrm{R}^{2}=0.9903\right)$, but have weaker mucoadhesive ability than FITC-chitosan $\left(\mathrm{WO}_{50}=91\right.$ $\left.\mathrm{mL}, \mathrm{R}^{2}=0.9970\right)$.

\subsection{Penetration into bladder mucosa}

In order to assess the penetration properties of NaFI-loaded conventional, PEG-Mal and PEGylated liposomes through bladder mucosa, fluorescence microscopy was employed. The liposome solutions were pipetted onto freshly excised porcine urinary bladder mucosa and were left in contact with the tissues for 15, 30, 45 and 60 minutes and were then frozen and sectioned. Fluorescent images were then collected and ImageJ software used to evaluate the penetration of liposomes. Figure 6 demonstrates that the PEG liposomes exhibit greater penetration ability $(\mathrm{p}<0.05)$ than conventional and PEG-Mal counterparts at all time points. The enhanced permeation performance of PEGylated liposomes into the mucosa, compared to conventional liposomes is in excellent agreement with the studies of PEGylated nanoparticles on different mucosal barriers (Wang et al., 2008; Mun et al., 2014). PEG provides stealth properties to liposomes, making them less interactive with biological tissues that facilitates their deeper penetration. This explains the greater diffusivity of PEGylated liposomes through mucosal epithelium compared to conventional liposomes. The maleimide-functionalised PEG liposomes are more mucoadhesive and will therefore form strong covalent bonds with thiols in mucosal tissue and hence their penetration is slightly retarded (Figure 6). Representative exemplary fluorescent images of the penetration of different liposomes through porcine bladder mucosa can be found in Supplementary Information (Figure S3). Better penetration of PEG liposomes into bladder mucosa could also provide some advantages for intravesical drug delivery; application of penetration enhancers such as dimethylsuphoxide to facilitate deeper anticancer drug penetration has previously been reported (Chen et al, 2003).

\subsection{In vitro release from liposomes}


The in vitro release studies for NaFI from conventional, PEG-Mal and PEGylated liposomes were conducted in $\mathrm{AU}$ solution at $37^{\circ} \mathrm{C}$ using a dialysis method and the cumulative release profiles are shown in Figure 7. Conventional liposomes exhibited a rapid release of NaFI, which reaches saturation after 2 h. PEGylated and PEG-Mal liposomes demonstrated a prolonged release, which reaches 95-100 $\%$ after 4 and $8 \mathrm{~h}$, respectively. This difference is clearly related to the presence of PEG on liposomal surfaces, which makes them more stable. A more prolonged release of a drug from PEG-Mal liposomes provides an advantage as it will ensure better efficiency and will maintain a therapeutically-relevant drug concentration in the bladder over a longer period of time following intravesical administration. A delayed release of NaFI from liposomes could also improve model drug retention on the bladder.

\section{Conclusion}

Three liposomal formulations were evaluated in this work for their retention in the urinary bladder, penetration into the mucosa and drug release in vitro. These formulations were prepared based on conventional liposomes, PEGylated liposomes and liposomes decorated with maleimidefunctionalised PEG. The liposomes with maleimide groups exhibited superior in vitro retention on the bladder tissue, which is related to their ability to form covalent bonds with thiols present in mucosal tissue. PEGylated liposomes were found to have a greater ability to penetrate deeper into the mucosal tissue due to the stealth character of PEG that facilitates mucus-penetrating properties.

\section{Acknowledgements}

The authors gratefully acknowledge the British Council Newton-Al-Farabi Partnership Programme, the Researcher Links Post-doctoral Mobility Grant (216046068) for financial support and for providing 2-years postdoctoral fellowship for Dr D.B. Kaldybekov at the University of Reading. Dr Reading) is thanked for access to fluorescence spectrometer and TEM. P.C. Turner Abattoirs (Farnborough, UK) is also acknowledged for providing pig bladders for experiments.

\section{Appendix A. Supplementary data}

\section{References}

Adamczak, M.I., Martinsen, Ø.G., Smistad, G., Hiorth, M., 2017. Polymer coated mucoadhesive liposomes intended for the management of xerostomia. Int. J. Pharm. 527, 72-78. 

604.

Barthelmes, J., Perera, G., Hombach, J., Dünnhaupt, S., Bernkop-Schnürch, A., 2011. Development of a mucoadhesive nanoparticulate drug delivery system for a targeted drug release in the bladder. Int. J. Pharm. 416, 339-345.

Barthelmes, J., Dünnhaupt, S., Unterhofer, S., Perera, G., Schlocker, W., Bernkop-Schnürch, A., 2013. Thiolated particles as effective intravesical drug delivery systems for treatment of bladder-related diseases. Nanomedicine 8, 65-75.

Berginc, K., Suljaković, S., Škalko-Basnet, N., Kristl, A., 2014. Mucoadhesive liposomes as new formulation for vaginal delivery of curcumin. Eur. J. Pharm. Biopharm. 87, 40-46.

Bernkop-Schnürch, A., 2005. Thiomers: The next generation of mucoadhesive polymers. Adv. Drug Deliv. Rev. 57, 1569-1582.

Brannigan, R.P., Khutoryanskiy, V.V., 2017. Synthesis and evaluation of mucoadhesive acryloylquaternized PDMAEMA nanogels for ocular drug delivery. Colloids Surfaces B Biointerfaces $155,538-543$.

Burjak, M., Bogataj, M., Velnar, M., Grabnar, I., Mrhar, A., 2001. The study of drug release from microspheres adhered on pig vesical mucosa. Int. J. Pharm. 224, 123-130.

Chuang, Y.C., Tyagi, P., Huang, C.C., Yoshimura, N., Wu, M., Kaufman, J., Chancellor, M.B., 2009. Urodynamic and immunohistochemical evaluation of intravesical botulinum toxin A delivery using liposomes. J. Urol. 182, 786-792.

Chuang, Y.C., Kaufmann, J.H., Chancellor, D.D., Chancellor, M.B., Kuo, H.C., 2014. Bladder instillation of liposome encapsulated onabotulinumtoxina improves overactive bladder symptoms: A prospective, multicenter, double-blind, randomized trial. J. Urol. 192, 1743-1749.

Chutipongtanate, S., Thongboonkerd, V., 2010. Systematic comparisons of artificial urine formulas for in vitro cellular study. Anal. Biochem. 402, 110-112.

Chen, D., Song, D., Wientjes, M.G., Au J. L-S., 2003. Effect of Dimethyl Sulfoxide on Bladder Tissue Penetration of Intravesical Paclitaxel. Clinical Cancer Research 9, 363-369.

Cook, M.T., Tzortzis, G., Charalampopoulos, D., Khutoryanskiy, V.V., 2011. Production and evaluation of dry alginate-chitosan microcapsules as an enteric delivery vehicle for probiotic bacteria. Biomacromolecules 12, 2834-2840.

Cook, M.T. Schmidt, Lee, S.A.E., Samprasit, W., Opanasopit, P., Khutoryanskiy, V.V., 2015. Synthesis of mucoadhesive thiol-bearing microgels from 2-(acetylthio)ethylacrylate and 2- 
hydroxyethylmethacrylate: novel drug delivery systems for chemotherapeutic agents to the bladder. J. Mater. Chem. B, 2015, 3, 6599-6604.

Davidovich-Pinhas, M., Harari, O., Bianco-Peled, H., 2009. Evaluating the mucoadhesive properties of drug delivery systems based on hydrated thiolated alginate. J. Control. Release 136, 38-44.

Davidovich-Pinhas, M., Bianco-Peled, H., 2011. Physical and structural characteristics of acrylated poly(ethylene glycol)-alginate conjugates. Acta Biomater. 7, 2817-2825.

Davidovich-Pinhas, M., Bianco-Peled, H., 2014. Methods to study mucoadhesive dosage forms, in: Khutoryanskiy, V.V. (Ed.), Mucoadhesive Materials and Drug Delivery Systems. John Wiley \& Sons, Ltd, pp. 175-196.

Frederik, P.M., Hubert, D.H.W., 2005. Cryoelectron microscopy of liposomes. Methods Enzymol. 391, 431-448.

GuhaSarkar, S., Banerjee, R., 2010. Intravesical drug delivery: Challenges, current status, opportunities and novel strategies. J. Control. Release 148, 147-159.

Harris, J.R., 1986. A comparative negative staining study of aqueous suspensions of sphingomyelin. Micron Microsc. Acta 17, 175-200.

Hombach, J., Bernkop-Schnürch, A., 2010. Mucoadhesive drug delivery systems, in: Schäfer-Korting, M. (Ed.), Drug Delivery. Springer Berlin Heidelberg, Berlin, Heidelberg, pp. 251-266.

Irmukhametova, G.S., Mun, G.A., Khutoryanskiy, V.V., 2011. Thiolated mucoadhesive and PEGylated nonmucoadhesive organosilica nanoparticles from 3-mercaptopropyltrimethoxysilane. Langmuir 27, 9551-9556.

Kandzija, N., Khutoryanskiy, V.V., 2017. Delivery of riboflavin-5'-monophosphate into the cornea: can liposomes provide any enhancement effects? J. Pharm. Sci. 106, 3041-3049.

Kawamorita, N., Yoshikawa, S., Kashyap, M., Tyagi, P., Arai, Y., Chancellor, M.B., Yoshimura, N., 2016. Liposome Based Intravesical Therapy Targeting Nerve Growth Factor Ameliorates Bladder Hypersensitivity in Rats with Experimental Colitis. J. Urol. 195, 1920-1926.

Khutoryanskiy, V.V., 2011. Advances in mucoadhesion and mucoadhesive polymers. Macromol. Biosci. 11, 748-764.

Kim, K., Kim, K., Ryu, J.H., Lee, H., 2015. Chitosan-catechol: A polymer with long-lasting mucoadhesive properties. Biomaterials 52, 161-170.

Kolawole, O.M., Lau, W.M., Mostafid, H., Khutoryanskiy, V.V., 2017. Advances in intravesical drug delivery systems to treat bladder cancer. Int. J. Pharm. 532, 105-117.

Kulkarni, S.B., Betageri, G. V, Singh, M., 1995. Factors affecting microencapsulation of drugs in liposomes. J. Microencapsul. 12, 229-246. 
Malmström, P.U., 2003. Intravesical therapy of superficial bladder cancer. Crit. Rev. Oncol. Hematol. 47, 109-126.

Mansfield, E.D.H., de la Rosa, V.R., Kowalczyk, R.M., Grillo, I., Hoogenboom, R., Sillence, K., Hole, P., Williams, A.C., Khutoryanskiy, V.V., 2016. Side chain variations radically alter the diffusion of poly(2-alkyl-2-oxazoline) functionalised nanoparticles through a mucosal barrier. Biomater. Sci. 35, 583-592.

Mun, E.A., Morrison, P.W.J., Williams, A.C., Khutoryanskiy, V.V., 2014. On the barrier properties of the cornea: A microscopy study of the penetration of fluorescently labeled nanoparticles, polymers, and sodium fluorescein. Mol. Pharm. 11, 3556-3564.

Mun, E.A., Williams, A.C., Khutoryanskiy, V.V., 2016. Adhesion of thiolated silica nanoparticles to urinary bladder mucosa: Effects of PEGylation, thiol content and particle size. Int. J. Pharm. 512, $32-38$.

Nii, T., Ishii, F., 2005. Encapsulation efficiency of water-soluble and insoluble drugs in liposomes prepared by the microencapsulation vesicle method. Int. J. Pharm. 298, 198-205.

Oswald, M., Geissler, S., Goepferich, A., 2016. Determination of the activity of maleimidefunctionalized phospholipids during preparation of liposomes. Int. J. Pharm. 514, 93-102.

Rangsimawong, W., Opanasopit, P., Rojanarata, T., Duangjit, S., Ngawhirunpat, T., 2016. Skin transport of hydrophilic compound-loaded PEGylated lipid nanocarriers: Comparative study of liposomes, niosomes, and solid lipid nanoparticles. Biol. Pharm. Bull. 39, 1254-1262.

Robenek, H., Severs, N.J., 2008. Recent advances in freeze-fracture electron microscopy: the replica immunolabeling technique. Biol. Proced. Online 10, 9-19.

Sasaki, H., Karasawa, K., Hironaka, K., Tahara, K., Tozuka, Y., Takeuchi, H., 2013. Retinal drug delivery using eyedrop preparations of poly-l-lysine-modified liposomes. Eur. J. Pharm. Biopharm. 83, 364-369.

Shtenberg, Y., Goldfeder, M., Schroeder, A., Bianco-Peled, H., 2017. Alginate modified with maleimide-terminated PEG as drug carriers with enhanced mucoadhesion. Carbohydr. Polym. 175, 337-346.

Sou, K., 2011. Electrostatics of carboxylated anionic vesicles for improving entrapment capacity. Chem. Phys. Lipids 164, 211-215.

Stewart, B.W., Wild, C.P., 2014. World cancer report 2014, International Agency for Research on Cancer.

Storha, A., Mun, E.A., Khutoryanskiy, V.V., 2013. Synthesis of thiolated and acrylated nanoparticles using thiol-ene click chemistry: towards novel mucoadhesive materials for drug delivery. RSC 
Adv. 3, 12275-12279.

492 Symonds, B., Lindsay, C.I., Thomson, N.R., Khutoryanskiy, V.V., 2016. Chitosan as a rainfastness 493 adjuvant for agrochemicals. RSC Adv. 6, 102206-102213.

494 Thompson, R.F., Walker, M., Siebert, C.A., Muench, S.P., Ranson, N.A., 2016. An introduction to 495 sample preparation and imaging by cryo-electron microscopy for structural biology. Methods 100, $496 \quad 3-15$.

497 Tonglairoum, P., Brannigan, R.P., Opanasopit, P., Khutoryanskiy, V.V., 2016. Maleimide-bearing 498 nanogels as novel mucoadhesive materials for drug delivery. J. Mater. Chem. B 4, 6581-6587.

499 Torre, L.A., Bray, F., Siegel, R.L., Ferlay, J., Lortet-tieulent, J., Jemal, A., 2015. Global Cancer $500 \quad$ Statistics, 2012. CA a cancer J. Clin. 65, 87-108.

501 Tyagi, P., Wu, P.C., Chancellor, M., Yoshimura, N., Huang, L., 2006. Recent advances in intravesical 502 drug/gene delivery. Mol. Pharm. 3, 369-379.

503 Verma, D.D., Verma, S., Blume, G., Fahr, A., 2003. Particle size of liposomes influences dermal 504 delivery of substances into skin. Int. J. Pharm. 258, 141-151.

505 Wang, Y.Y., Lai, S.K., Suk, J.S., Pace, A., Cone, R., Hanes, J., 2008. Addressing the PEG 506 mucoadhesivity paradox to engineer nanoparticles that "slip" through the human mucus barrier. 507 Angew. Chemie - Int. Ed. 47, 9726-9729.

508 509 
$510 \quad$ Figures and legends

511

512

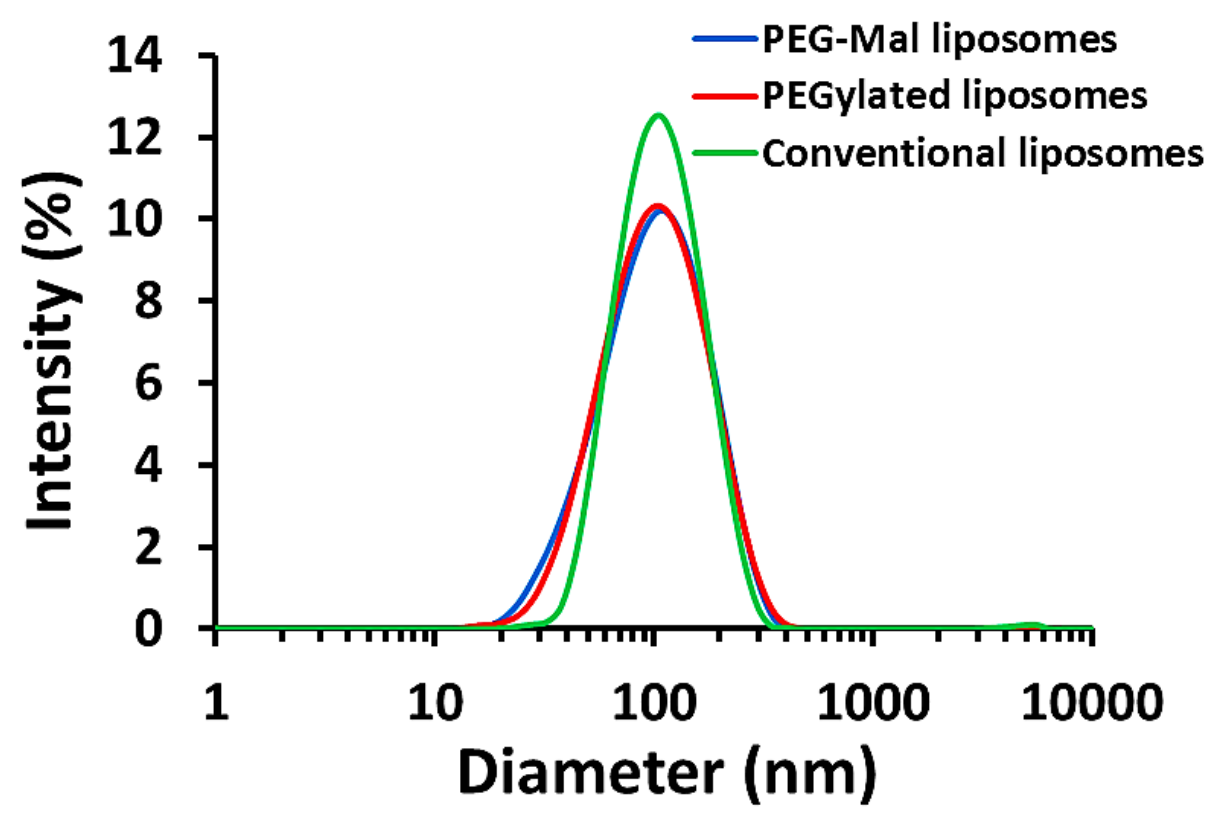

513

514 Figure 1

515

516

517

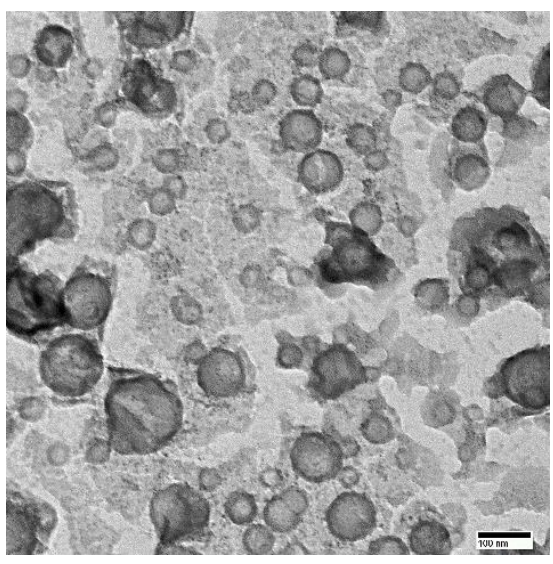

A

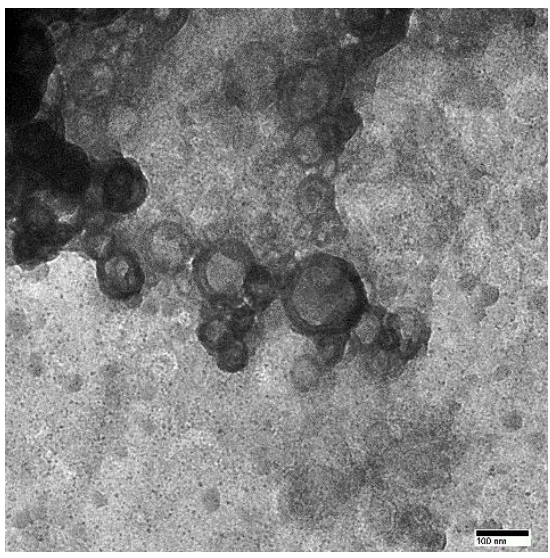

B

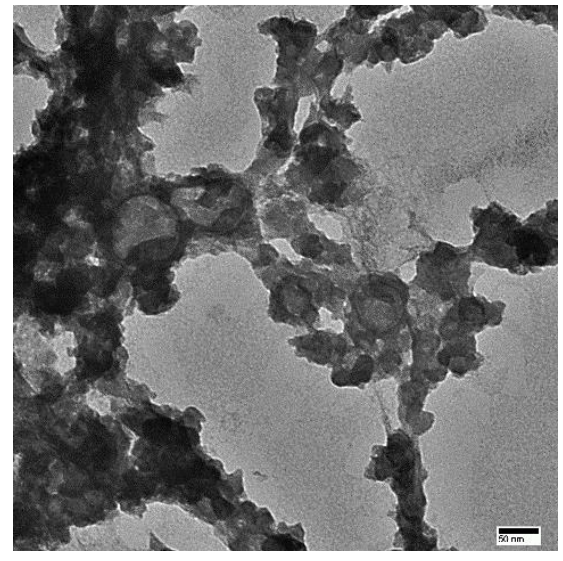

C

518

519 Figure 2

520

521 
FITC-chitosan
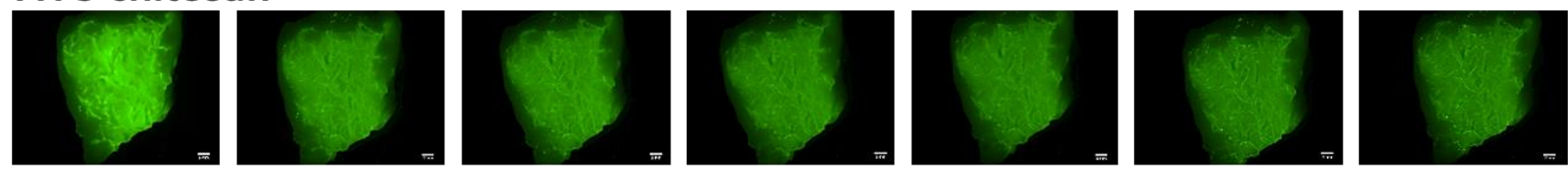

\section{PEG-Mal liposomes}
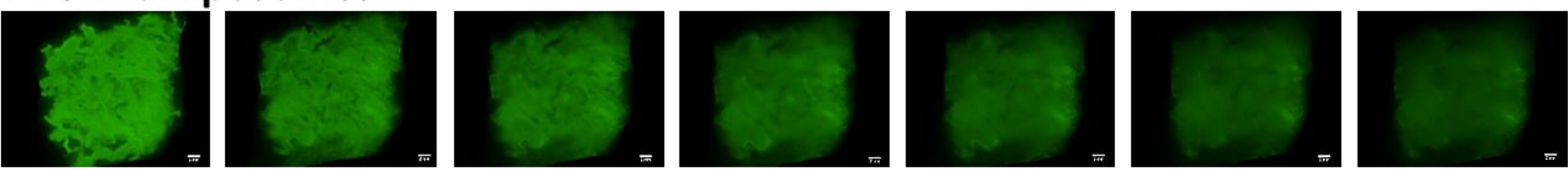

\section{PEGylated liposomes}
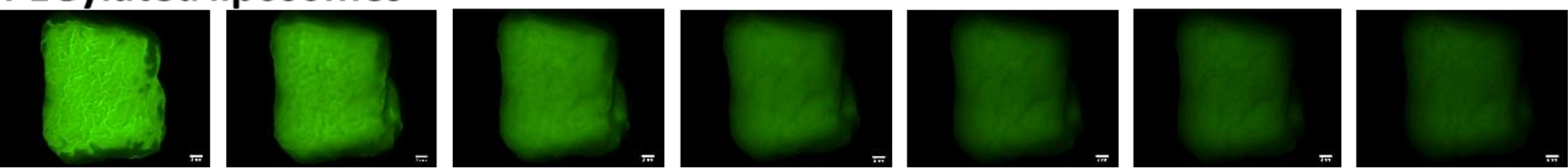

Conventional liposomes
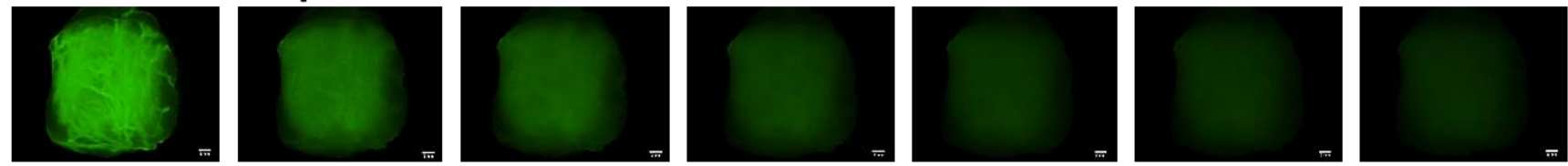

\section{FITC-dextran}
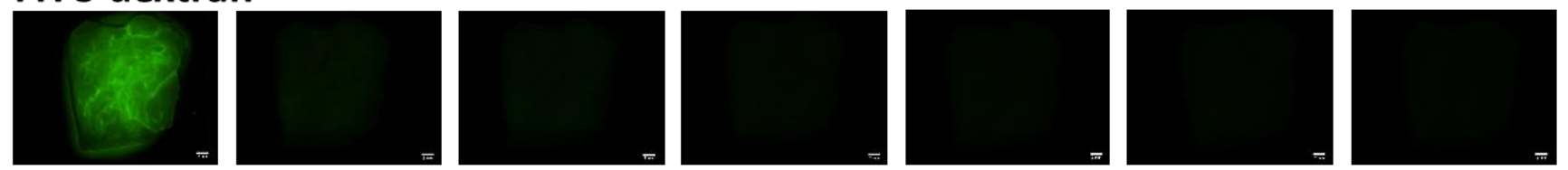

0

10

20

60

80

$100 \mathrm{~mL}$

522

523

524 Figure 3

525

526 


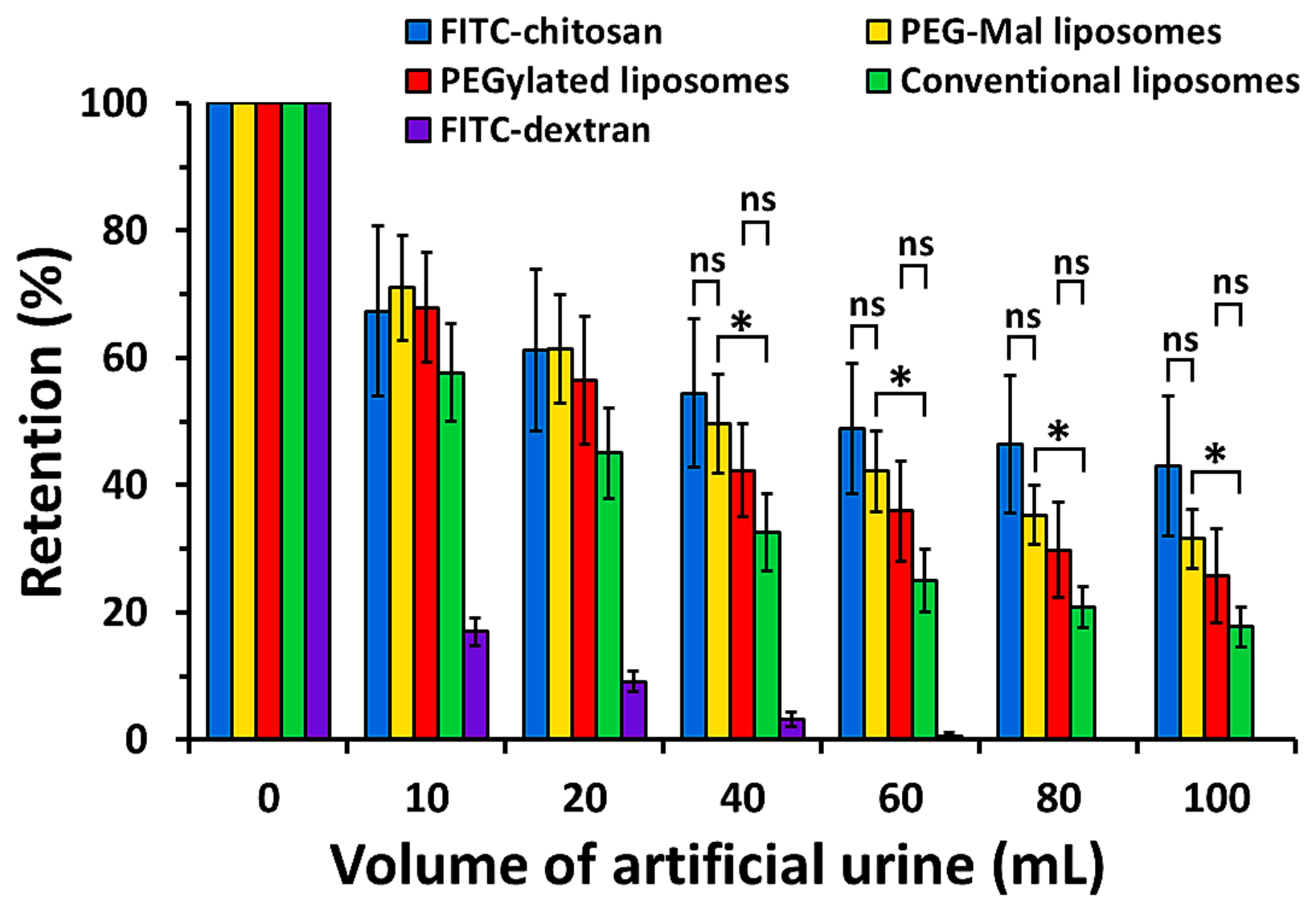

527

528

529 Figure 4

530

531 


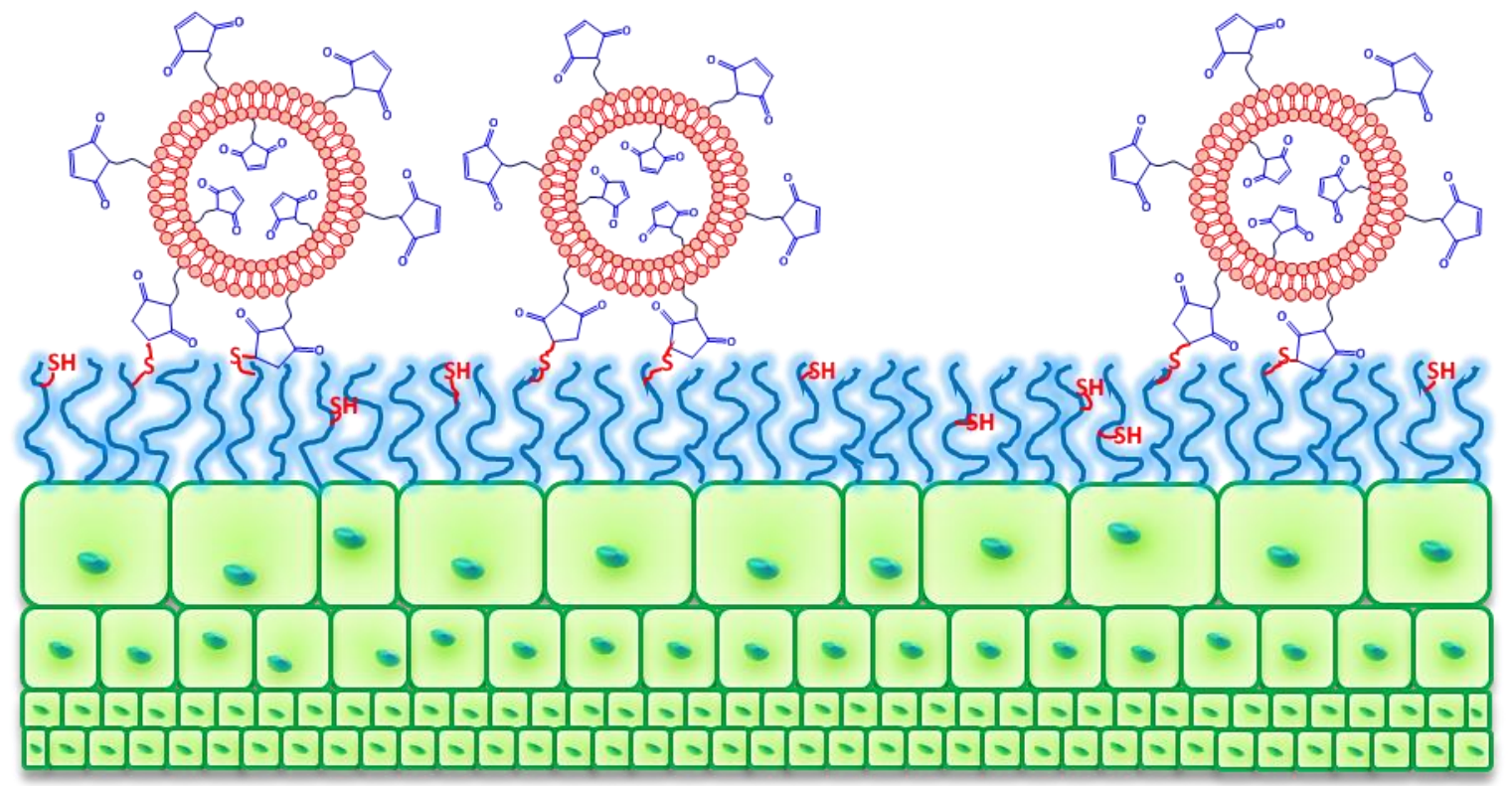

533

534 Figure 5

535

536 
Conventional liposomes $\square$ PEGylated liposomes $\square$ PEG-Mal liposomes

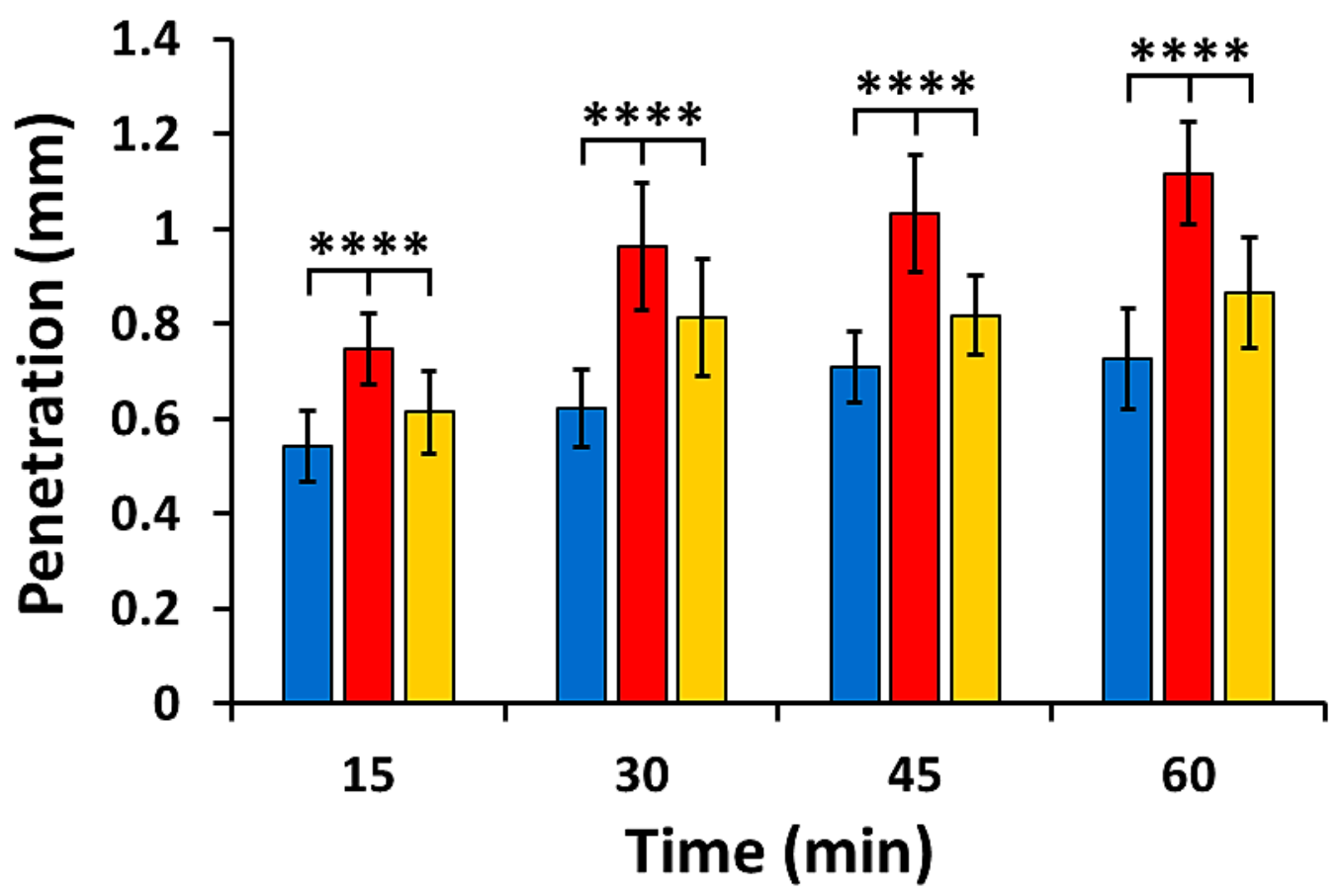

538

539 Figure 6

540 
542

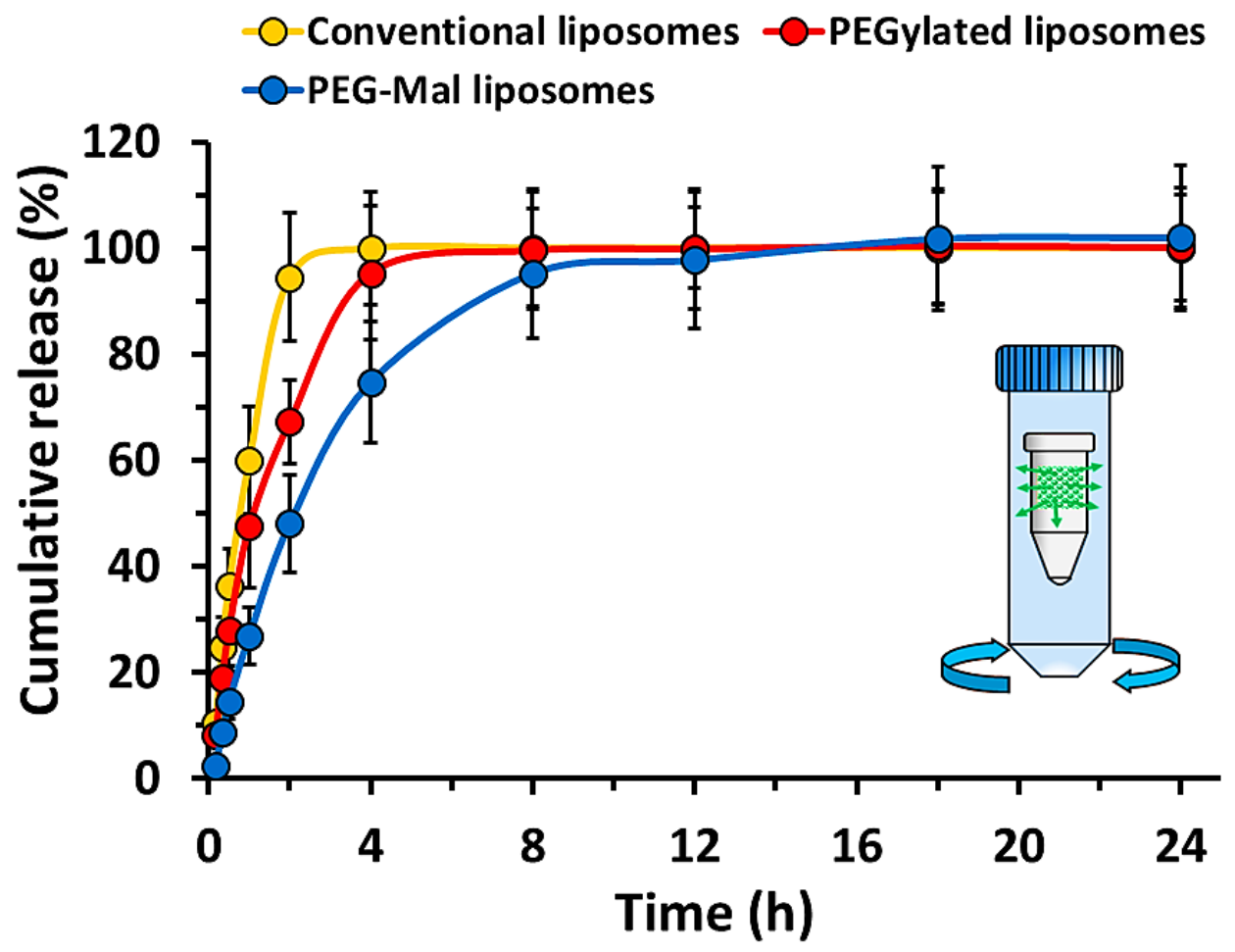

543

544 Figure 7

545

546 
547 Figure 1 Size distribution of conventional, PEGylated and PEG-Mal liposomes as determined by DLS 548

549 Figure 2 TEM micrographs of conventional (A), PEGylated (B) and PEG-Mal liposomes (C). Scale 550 bars are $100 \mathrm{~nm}$ for (A) and (B), and $50 \mathrm{~nm}$ for (C) 551

552 Figure 3 Exemplar fluorescence images showing retention of FITC-chitosan, PEGylated, PEG-Mal 553 liposomes, conventional liposomes and FITC-dextran on porcine urinary bladder mucosa washed with 554 different volumes of AU. Scale bars are $2 \mu \mathrm{m}$ 555

556 Figure 4 Percentage retention of conventional liposomes, PEGylated, PEG-Mal liposomes, FITC557 chitosan and FITC-dextran on porcine urinary bladder mucosa after irrigating with different volumes of 558 AU. Data are expressed as mean \pm standard deviation $(\mathrm{n}=3)$. *Statistically significant difference $(\mathrm{p}<$ $559 \quad 0.05)$

560

561 Figure 5 Proposed mechanism of bonding between maleimide-functionalised liposomes and mucosal 562 surfaces

563

564 Figure 6 Penetration of the conventional, PEGylated, PEG-Mal liposomes over 60 mins. Values 565 represent the mean penetration across 10 separate porcine urinary bladder tissue sections \pm standard 566 deviation

568 Figure 7 Cumulative release profile of fluorescein sodium from liposomal formulations. Data expressed 569 as mean standard deviation $(n=3)$. Insert shows the experimental set-up used in the release studies

570

571 
572 Table 1 The composition (\%) of lipid nanocarrier formulations.

\begin{tabular}{llllll}
\hline Liposome formulations & $\mathrm{PC}$ & $\mathrm{CHO}$ & $\mathrm{MPEG}_{2000}-\mathrm{DSPE}$ & $\mathrm{PEG}_{2000}-\mathrm{DSPE}-\mathrm{Mal}$ & $\mathrm{NaFI}$ \\
\hline Conventional & 0.773 & 0.077 & - & - & 0.2 \\
PEGylated & 0.773 & 0.077 & 0.075 & - & 0.2 \\
PEG-Mal & 0.773 & 0.077 & - & 0.075 & 0.2
\end{tabular}

PC - Soybean L-alpha-phosphatidylcholine; CHO - Cholesterol; MPEG2000-DSPE - [N-(carbonylmethoxypolyethylene glycol-2000)-1,2-distearoyl-sn-glycero-3-phosphoethanol-amine, sodium salt]; $\quad \mathrm{PEG}_{2000}$-DSPE-Mal _ $\quad$ 1,2-distearoyl-sn-glycero-3-phosphoethanolamine-N[maleimide(polyethylene glycol)-2000] ammonium salt; NaFI - Fluorescein sodium salt

573

574

575

576

\begin{tabular}{lllllll}
\hline $\begin{array}{l}\text { Liposome } \\
\text { formulations }\end{array}$ & $\begin{array}{l}\text { Mean diameter, } \\
\mathrm{nm}\end{array}$ & $\mathrm{PDI}$ & $\begin{array}{l}\text { Zeta potential, } \\
\mathrm{mV}\end{array}$ & \%EE & $\% \mathrm{LC}$ & $\mathrm{WO}_{50}, \mathrm{~mL}$ \\
\hline Conventional & $97 \pm 1$ & 0.145 & $-53 \pm 1$ & $53 \pm 6$ & $12 \pm 1$ & 15 \\
PEGylated & $85 \pm 1$ & 0.217 & $-32 \pm 2$ & $27 \pm 2$ & $6 \pm 1$ & 24 \\
PEG-Mal & $86 \pm 1$ & 0.224 & $-37 \pm 1$ & $25 \pm 2$ & $5 \pm 1$ & 48
\end{tabular}

$\mathrm{WO}_{50}$, volume of AU required to wash out $50 \%$ of liquid formulation. Results are given as mean \pm standard deviation $(\mathrm{n}=3)$ 